\title{
On the effects of roughness on the nonlinear dynamics of a bolted joint: a multiscale analysis
}

\author{
J. Armand ${ }^{1, *}$ L. Salles ${ }^{1}$, C.W.Schwingshackl ${ }^{1}$, D.Süß ${ }^{2}$, K.Willner $^{2}$ \\ 1 Imperial College London \\ South Kensington Campus, London, SW7 2AZ, UK \\ 2 Friedrich-Alexander-Universitaet Erlangen-Nuernberg \\ Egerlandstrasse 591058 Erlangen, Germany
}

\begin{abstract}
Accurate prediction of the vibration response of friction joints is of great importance when estimating both the performance and the life of build-up structures. The contact conditions at the joint interface, including local normal load distribution and contact stiffness, play a critical role in the nonlinear dynamic response. These parameters strongly depend on the mating surfaces, where the surface roughness is well known to have a significant impact on the contact conditions in the static case. In contrast, its effects on the global and local nonlinear dynamic response of a build-up structure is not as well understood due to the complexity of the involved mechanisms. To obtain a better understanding of the dependence of the nonlinear dynamic response on surface roughness, a newly proposed multiscale approach has been developed. It links the surface roughness to the contact pressure and contact stiffness, and in combination with a multiharmonic balance solver, allows to compute the nonlinear dynamic response for different interface roughness. An application of the technique to a single bolted lap joint highlighted a strong impact of larger roughness values on the pressure distribution and local contact stiffness and in turn on the nonlinear dynamic response.
\end{abstract}

Keywords: bolted joint, elastic rough contact, nonlinear dynamics

\footnotetext{
* Corresponding author.

E-mail address : j.armand13@imperial.ac.uk.

Tel.: +44(0)2075942243
} 


\section{Introduction}

Engineering structures usually comprise many components that are often connected together via bolted joints and interact through contacting interfaces. Under dynamic excitation, forces occurring at these contact interfaces can have a significantly nonlinear character. At low excitation amplitudes, most of the joint surface remains stuck and behaves linearly. At higher excitation amplitudes, parts or all of the joint surface experiences stick-slip transitions which may lead to a significant softening effect in the dynamic response, as well as some energy dissipation due to friction resulting in an increased damping and wear of the components $[1,2,3]$.

The understanding of the joint behaviour is greatly driven by the experimental characterisation of the joints $[4,5,6,7,8,2,1]$, leading to a great diversity in the treatment of joint models depending on the application and the computational power at hand. Some examples include detailed finite element models of joint components $[9,10,11]$, continuum element approaches using zero-thickness elements $[12,3]$ and thin-layer elements $[13,14,15]$, lumped models $[6,16,17]$, whole-joint modelling using hysteresis elements $[18,19]$, and node-to-node coupling with friction contact elements [20, 21, 22, 23, 24]. In the latter modelling approach a number of friction elements is introduced across the contact area in order to capture its nonlinear behaviour. Each friction element is based on a modified Coulomb model and is characterised by: (i) normal load, (ii) friction coefficient (iii) normal and (iv) tangential contact stiffness. Currently the values of the contact stiffnesses are assumed to be uniform across the contact area in all node-to-node approaches, although previous works $[25,26]$ showed that these values were dependent on the loading conditions. This dependency can lead to a variation of the localised contact stiffness with regards to the initial contact condition at the interface, but also during a vibration cycle. Given the often high sensitivity of frictional joints towards the contact stiffness $[27,28]$, this variation can be expected to have a significant impact on the nonlinear dynamic response and must be considered during analysis.

In the present paper, a new multiscale approach is proposed to compute the local values of the contact stiffnesses depending on surface roughness and loading conditions, and evaluate the impact on the dynamic behaviour of an assembled structures. Two separate space scales are used: a macro-scale for the dynamic analysis, and a micro-scale for the contact analysis which allows a better discretisation of the contact area and hence a higher accuracy. The proposed approach will be described in detail before applying it to a single bolted joint to demonstrate the impact of surface roughness on the local contact conditions and in turn on the nonlinear dynamic response of the system. It is worth noting that in this study, the contact is assumed to remain elastic, i.e. the asperities do not deform plastically and no plastic flow occurs underneath the contact surfaces.

\section{Current nonlinear dynamic modelling approach}

A previously developed nonlinear dynamic analysis code FORSE (FOrced Response SuitE) will be used in this investigation. FORSE is based on the multiharmonic representation of the steady-state response and allows large scale realistic friction interface modelling. The main features of the methodology can be found in $[29,30,31,21,32]$ and only an overview of the analysis will be presented here to provide the necessary background information.

The equation of motion of a frictional joint can be expressed as a linear part, which is independent of the vibration amplitudes, and a nonlinear part resulting from friction at the interface. It can be written in the following form:

$$
\mathbf{M} \ddot{\mathbf{q}}(t)+\mathbf{C} \dot{\mathbf{q}}(t)+\mathbf{K q}(t)+\mathbf{f}[\mathbf{q}(t), \dot{\mathbf{q}}(t)]=\mathbf{p}(t)
$$

where $\mathbf{q}(t)$ is a vector of displacements for all degrees of freedom (DOFs) in the system; $\mathbf{K}, \mathbf{C}$, and $\mathbf{M}$ are stiffness, damping, and mass matrices of the linear model; $\mathbf{f}[\mathbf{q}(t), \dot{\mathbf{q}}(t)]$ is a vector of nonlinear, friction interface forces, which is dependent on displacements and velocities of the interacting nodes; and $\mathbf{p}(t)$ is a vector of periodic exciting forces. The variation of the displacements in time is represented by a restricted 


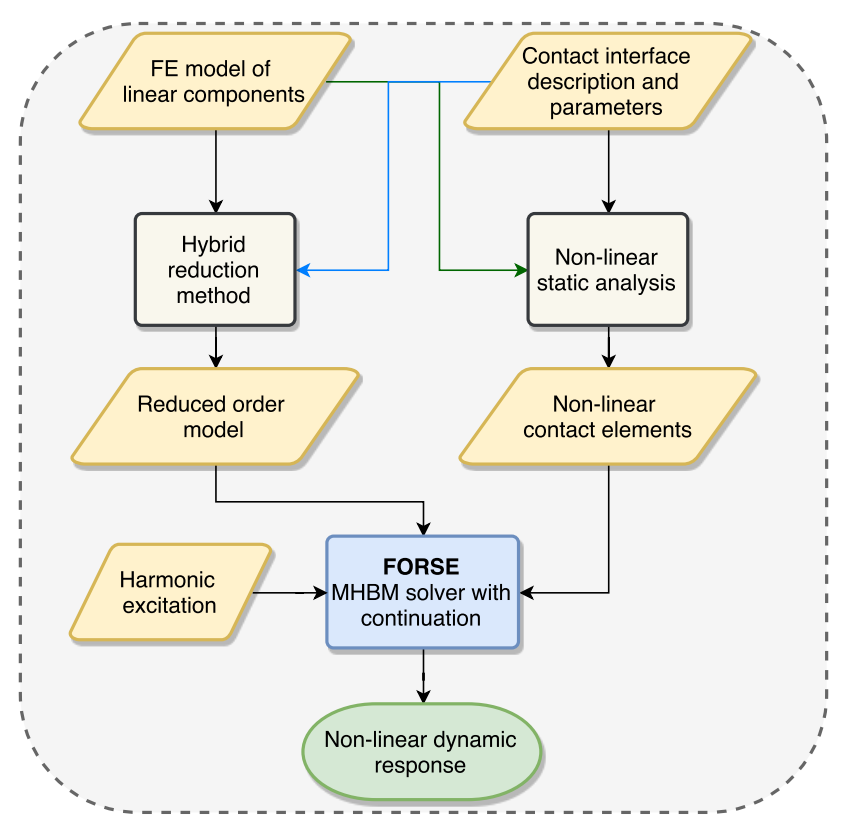

Figure 1: Scheme of the forced response analysis

Fourier series, which can contain as many harmonic components as necessary to approximate the solution,

$$
\mathbf{q}(t)=\mathbf{Q}_{0}+\sum_{j=1}^{n}\left(\mathbf{Q}_{j}^{c} \cos m_{j} \omega t+\mathbf{Q}_{j}^{s} \sin m_{j} \omega t\right)
$$

where $\mathbf{Q}_{j}^{c, s}$ are vectors of harmonic coefficients for the system degrees of freedom (DOFs); $n$ is the number of harmonics that are used in the multiharmonic displacement representation; and $\omega$ is the principal vibration frequency. The calculations performed with the code to obtain a nonlinear dynamic response are summarised in the flowchart in Fig. 1. The linear representation of the structure is based on a finite element model, but in order to model the nonlinear interactions at the contact interface, the contact interface elements developed in [31] are being used.

In the current modelling approach, a detailed FE model of the system is used to extract the modal properties required for the model. In order to reduce the size of the problem and therefore its computational cost, a hybrid reduction method developed by Petrov [32,33] is then applied. The matching FE nodes at the two contact interfaces are then connected via nonlinear contact elements [31] to form an assembled structure for the nonlinear dynamic analysis. Each friction element, depicted in Fig. 2, comprises two coupled Jenkins elements [34] allowing for 2D in-plane motion, and a third spring element allowing for normal load variation. Four parameters characterize its properties: the friction coefficient $\mu$, the initial normal load $N_{0}$, and the tangential $k_{t}$ and normal $k_{n}$ contact stiffness. The values of $\mu, k_{n}$ and $k_{t}$ are usually determined experimentally $[35,36,37]$ and are assumed to remain constant throughout the analysis. The initial normal load $N_{0}$ at the interface is usually obtained via a nonlinear quasi-static analysis using a FE software. The final nonlinear algebraic system of the resulting reduced model in the frequency domain can then be represented as

$$
\tilde{\mathbf{Q}}=A(\omega)\left(\tilde{\mathbf{F}}-\tilde{\mathbf{F}}_{\mathrm{nl}}(\tilde{\mathbf{Q}})\right),
$$

where $\tilde{\mathbf{Q}}$ is the vector of the Fourier coefficients of the displacements at the interface, $A(\omega)$ the frequency response, $\tilde{\mathbf{F}}$ the vector of the Fourier coefficients of the excitation force and $\tilde{\mathbf{F}}_{\mathrm{nl}}$ the vector of the Fourier coefficients of the nonlinear contact forces. This nonlinear system is solved by means of an alternating frequency time procedure (AFT) $[38,39]$ depicted in Fig. 3 where $\mathbf{z}$ denotes the tangential slips. DFT and iDFT refer to the Discrete Fourier Transform and the inverse Discrete Fourier Transform, respectively. $\boldsymbol{f}_{\text {pre }}$ 


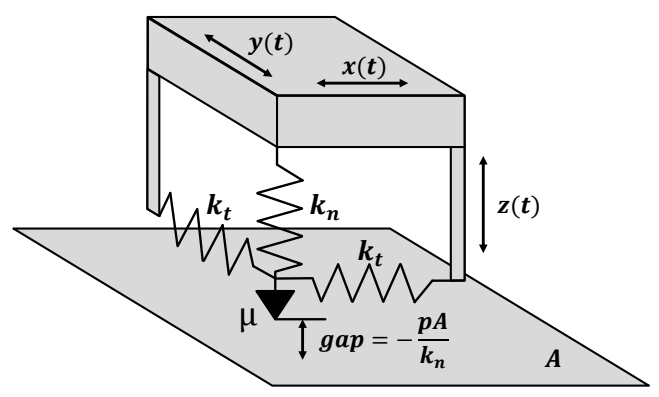

Figure 2: 3D nonlinear contact element

is the prediction of the nonlinear contact forces in time domain based on a penalty method and $\boldsymbol{f}_{\text {cor }}$ is the correction of these contact forces to ensure the frictional contact laws are respected.

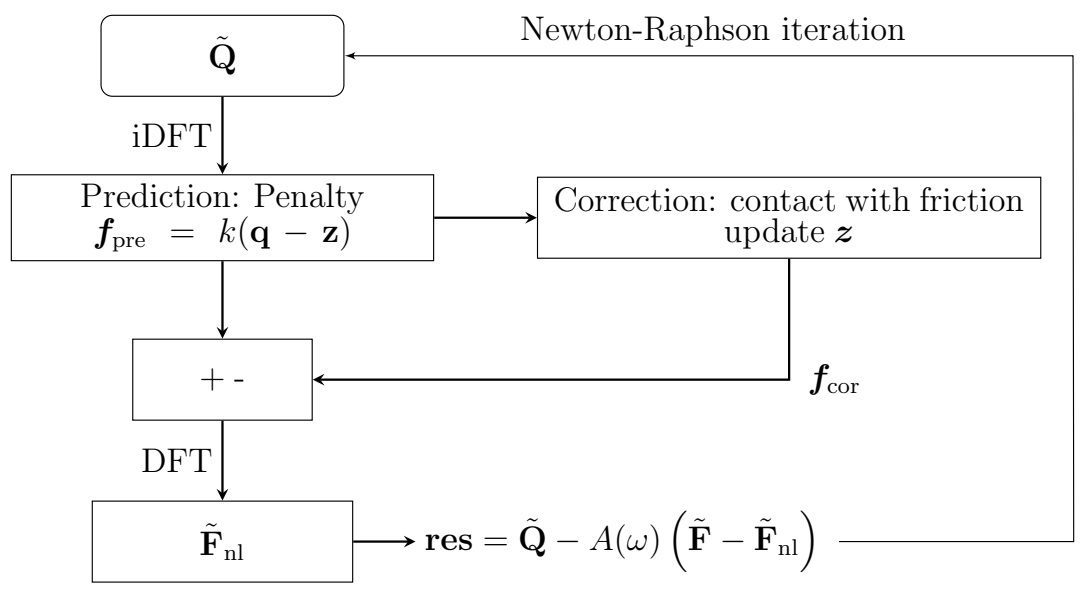

Figure 3: Scheme of the AFT procedure

The analysis results in a nonlinear frequency response function of the structure, together with detailed information about the contact conditions and energy dissipation at the interface nodes.

\section{Multiscale approach for variable contact stiffness}

As discussed earlier, it is necessary to account for variable contact stiffness across the contact area in order to obtain a more accurate prediction of the nonlinear response of a friction joint system. In the multiscale approach that is proposed here, the local contact stiffness is computed using a Boundary-Element -Method-based contact solver previously developed and validated by the authors [40, 41].

\subsection{A contact solver based on the Boundary-Element-Method (BEM)}

The contact solver uses the projected conjugate gradient method [42] and a discrete-convolution fast Fourier transform to accelerate the computation. It assumes the elastic half-space body description, which makes it possible to use the Boussinesq and Cerruti potentials $[43,44]$ to compute the surface elastic 
deflections in the normal and tangential directions from the pressures and shear tractions in the contact area. Equation 4 gives the component of normal displacement $u_{z}$ due to a pressure distribution $p$.

$$
u_{z}(x, y)=\frac{1-\nu^{2}}{\pi E} \int_{-\infty}^{+\infty} \int_{-\infty}^{+\infty} \frac{p(\xi, \eta)}{\sqrt{(\xi-x)^{2}+(\eta-y)^{2}}} d \xi d \eta
$$

where $E$ and $\nu$ are the Young's modulus and Poisson ratio of the material, respectively. Equation 5 is the discretised form of Eq. 4 on a regular grid of $N_{x} \times N_{y}$ points.

$$
u_{z}(i, j)=K_{z z} \otimes p=\sum_{k=1}^{N_{x}} \sum_{l=1}^{N_{y}} p(k, l) K_{z z}(i-k, j-l)
$$

where $\otimes$ denotes the discrete convolution product and $K_{z z}(i, j)$ are the discrete influence coefficients [41] that give the normal displacement resulting from unit pressure on the element centred on the grid point $(i, j)$. Similar equations are used to compute the the tangential displacements. The first step of the algorithm is to solve the normal contact problem using the conjugate gradient method, after which the tangential problem can be solved using the Coulomb friction law to bound the shear distribution in the slipping region.

\subsection{Multiscale approach for contact stiffness computation}

The combination of the nonlinear dynamic analysis in FORSE (see section 2) and the BEM-based contact solver described in the previous section was originally proposed for a multi-scale approach to evaluate the influence of wear on the nonlinear dynamic response [41]. Significant modifications and refinements were necessary to allow an accurate computation of contact stiffnesses for rough surfaces using the BEM-based contact solver. It was necessary to compute the normal load, the initial gap as well as the normal and tangential contact stiffnesses very accurately to provide improved input data to the friction elements used in the nonlinear dynamic analysis.

Initially a refined contact analysis is performed using a BEM-based contact solver on the whole contact interface (see Fig. 5). The applied loading is the total normal load transmitted through the contact area, which in case of a bolted joint corresponds to the tightening force of the bolt. The result of this analysis is a normal load and gap distribution which can be used to define all the 3D nonlinear contact elements used in FORSE (see Fig. 9). In a next step a separate contact analysis is performed over the area of every nonlinear contact element, using the previously extracted local normal loads. This analysis allows the computation of the characteristic 'normal load - indentation' curve, from which a localised normal contact stiffness $k_{n}$ can be extracted. At this stage in the process, the tangential load transmitted through the contact is not known and the tangential stiffness $k_{t}$ cannot be extracted from the contact solver yet. An initial $k_{t}$ value must therefore be assumed, which is based on Mindlin [45]:

$$
\frac{k_{t}}{k_{n}}=\frac{2(1-\nu)}{(2-\nu)}
$$

where $\nu$ is the Poisson ratio of the material.

Using this initial set of parameters defining the 3D contact elements, namely the normal load $N$, the gap $g$, the measured friction coefficient $\mu$ and the computed normal and tangential contact stiffness $k_{n}$ and $k_{t}$ for each element, a nonlinear dynamic analysis is conducted in FORSE. The dynamic loads transmitted through the contact over one vibration cycle at resonance are then extracted from that analysis. These dynamic loads are in a next step used as new inputs for the contact solver. Now that the tangential loads are available as well, the frictional hysteresis loop can be computed for each nonlinear element, from which the tangential contact stiffness can be extracted, as described in Fig. 4.

It is worth noting that the current nonlinear friction model implemented in FORSE does not allow the use of contact stiffnesses varying with the normal load, which is why the used contact stiffnesses are computed for the average normal load obtained over one vibration cycle. 


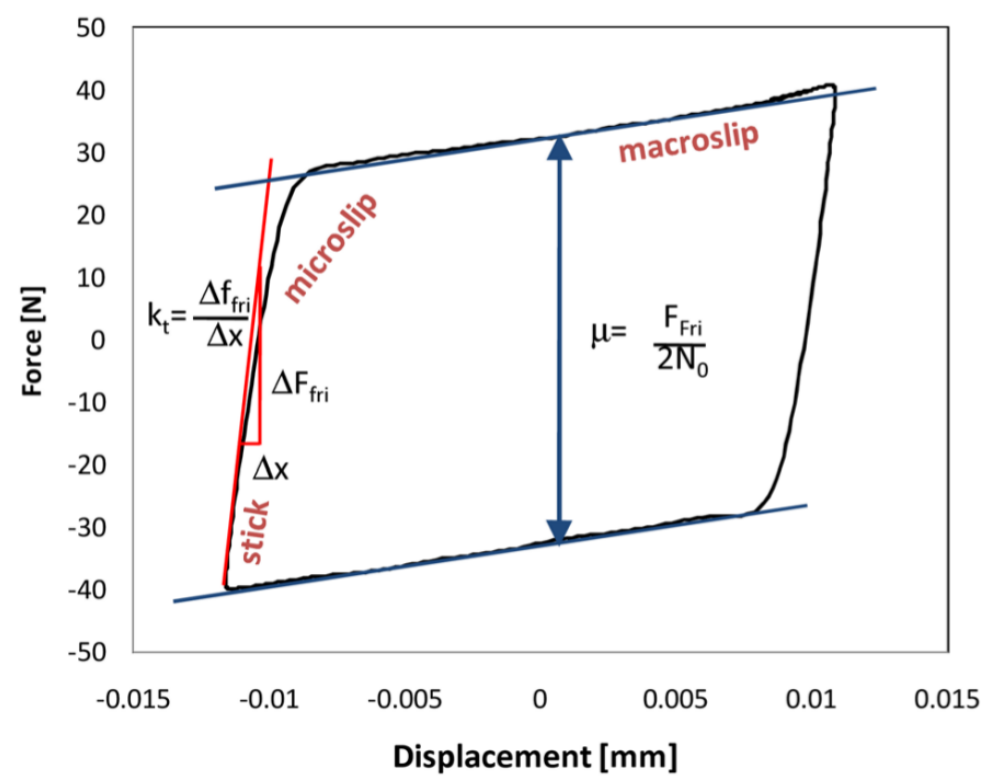

Figure 4: Frictional hysteresis loop and definition of tangential contact stiffness $k_{t}[27]$

\section{Application to a resonator system with smooth contact interface}

The impact of a normal load dependent contact stiffness on the dynamic response will be investigated on a lap joint with a single bolt, depicted in Fig. 6, and previously proposed and studied by Süß and Willner [3, 46]. The setup consists of an oscillator which is made of stainless steel and primarily consists of two masses which are connected through a bending spring and a bolted lap joint. As both parts of the structure are monolithic, the only joint in the system is the central bolted joint which can be investigated in an isolated manner.

\subsection{Linear finite element model}

A full three-dimensional finite element model (see Fig. 6) has been generated in Abaqus with linear hexahedral elements for both masses (19504 elements) and the bolt (7830 elements). The bolt is rigidly connected to each mass via "tie constraints". Particular care has been taken to refine the matching meshes at the contact interfaces to allow the introduction of a sufficient number of friction elements for the nonlinear analysis. This fine discretisation of the contact is required to capture local microslip effects which play a critical role in the energy dissipation of the system. All linear components of the system have the same material properties which are a Young's modulus of 198, 400 [MPa], a Poisson ratio of 0.3 and a density of $7,949[\mathrm{~kg} / \mathrm{m} 3]$.

\subsection{Nonlinear contact model}

In order to observe the nonlinear dynamic behaviour of the studied system, and more precisely the first breathing mode of the leaf spring (with motion occuring only along $x$ direction) depicted in Fig. 7, an initial nonlinear dynamic analysis was performed. In this analysis, a number of 3D nonlinear contact elements has been introduced at the contact interface. Each friction element connects a linear finite element node from one side of the contact to its matching node on the opposing side of the contact and transmits the nonlinear forces. A convergence analysis, based on the resulting FRFs, showed that 340 friction elements were sufficient to obtain a converged dynamic response for the mode of interest, which is the first bending mode of the bending spring shown in Fig. 6. This number of friction elements has been used in all subsequent analyses presented in this paper. Similarly, the use of the first three harmonics, plus the harmonic 'zero' in the Fourier truncated representation of the response was shown to be sufficient. 


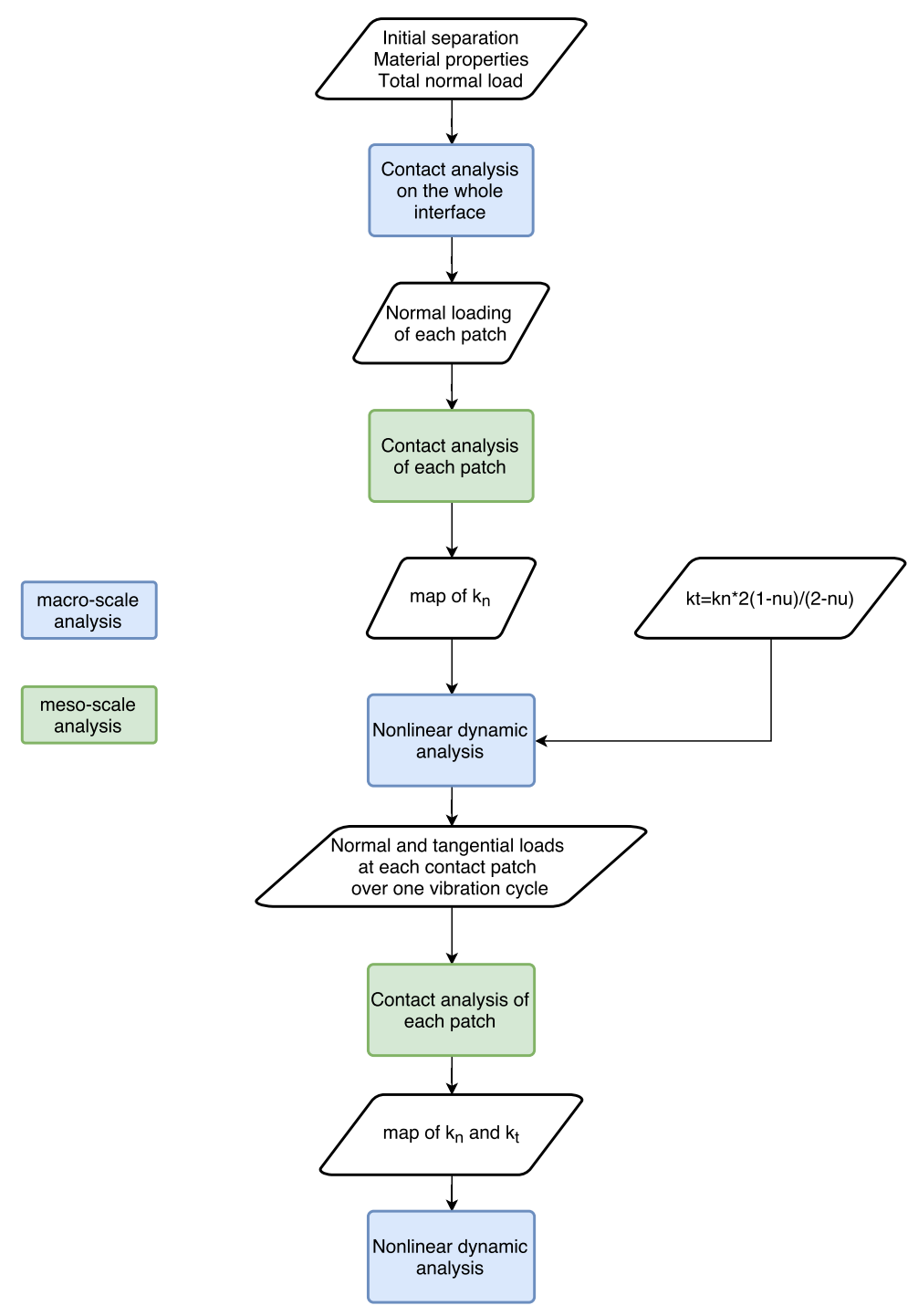

Figure 5: Scheme of the multiscale analysis

For this initial analysis, the contact surface of the joint is supposed to be perfectly flat and smooth. A nonlinear static analysis performed in Abaqus with a $30 k N$ pretension load of the bolt is providing the normal load required for the definition of each friction element used in the nonlinear dynamic analysis. The pre-tensioning of the bolt is applied by using the bolt load feature available in Abaqus. The shaft of the bolt is cut at mid-length through a pre-tension section, and the prescribed force is applied to this section along the axis of the bolt. A surface-to-surface approach with a direct enforcement method (based on Lagrange multipliers) is then used to solve the contact problem. A penalty method formulation with a friction coefficient $\mu=0.6$ is used to describe the frictional behaviour. The resulting pressure distribution is shown in Fig. 10. As expected, the pressure is maximum around the bolt hole and reduces concentrically leading to an area of lower compression on the corners of the contact interface.

For the baseline modelling approach, the normal and tangential contact stiffnesses of each contact element are set to $6 e 4\left[\mathrm{~N} / \mathrm{mm}^{3}\right]$ based on previous measurements reported in [30] to represent the current best practice. For the multiscale approach model, these values are replaced by the ones computed using the BEM-based contact solver, as described in section 3. For the initial comparison, only the normal contact 


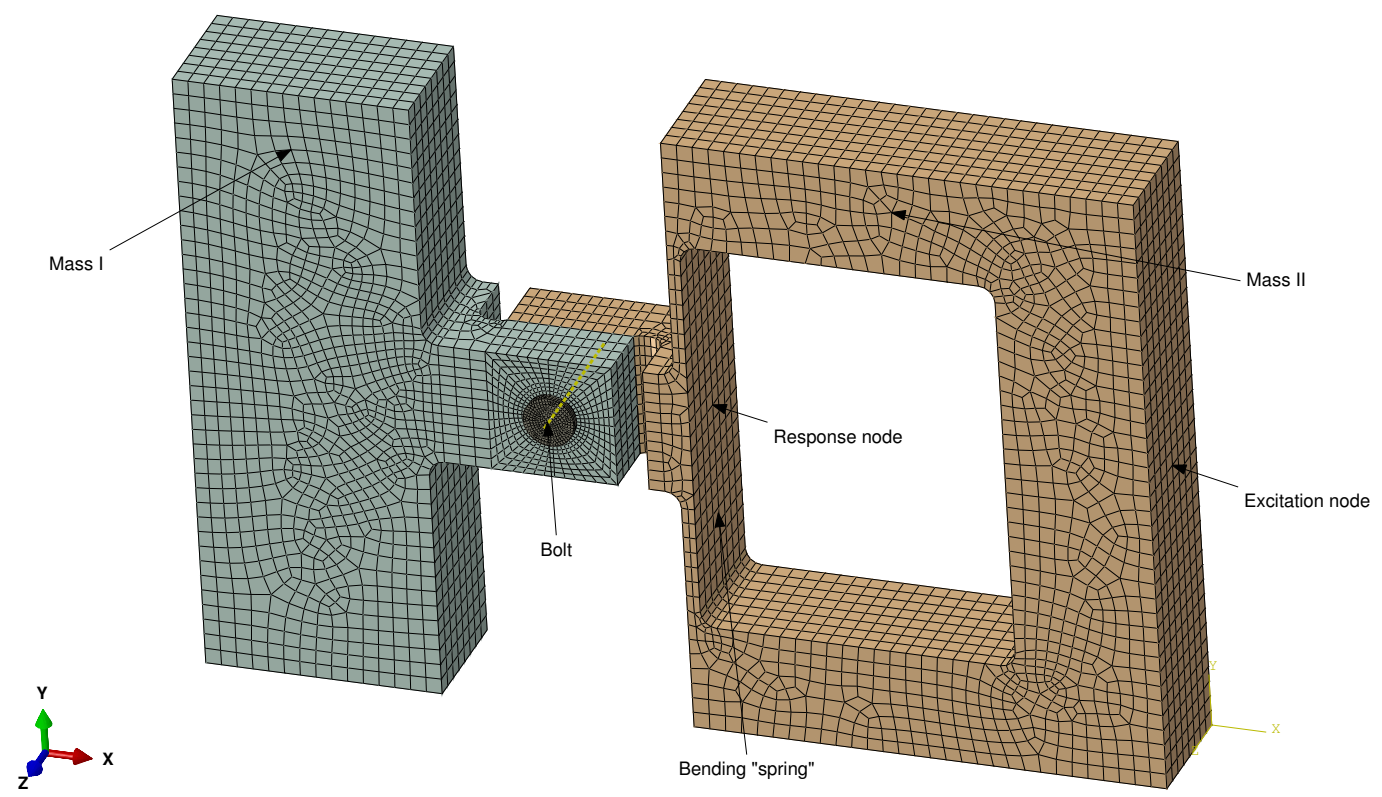

Figure 6: Finite Element model of the resonator

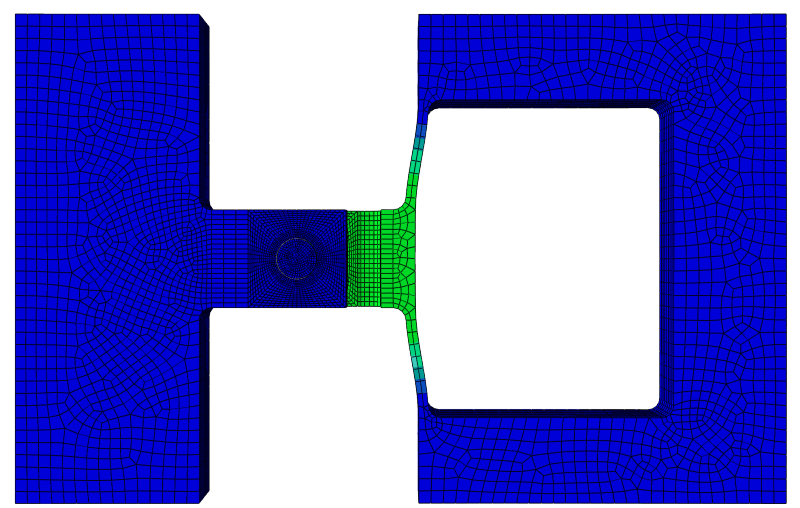

Figure 7: Deformed shape of the first breathing mode of the leaf spring

stiffnesses were extracted from the contact solver and the tangential contact stiffnesses were assumed to be equal to the normal ones. Figure 8 shows the values of normal contact stiffness $k_{n}$ computed using the contact solver. It can be seen that these values are consistent with the pressures shown in Fig. 12: the higher the pressure, the higher the normal contact stiffness. Compared to the uniform value of $6 e 4 \mathrm{~N} / \mathrm{mm} 3$ ] used in the current modelling approach, the computed values of $k_{n}$ vary greatly across the contact area between 0 (where there is no contact) and 1.74e5 [N/mm3] around the hole where the pressure is maximum. Interestingly, the average value of $k_{n}$ is $6.6 e 4[\mathrm{~N} / \mathrm{mm} 3]$, which is very close to the measured reference value of $6 e 4[\mathrm{~N} / \mathrm{mm} 3]$. 


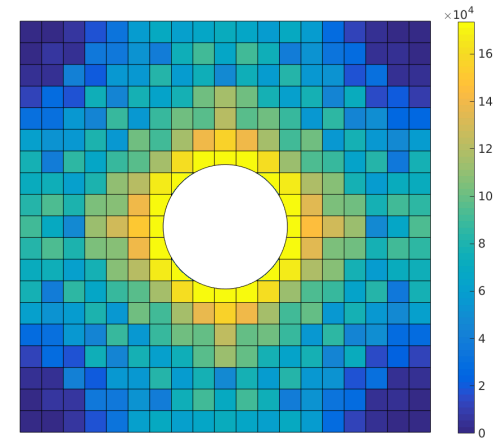

Figure 8: Distribution of normal contact stiffness $k_{n}$ in $[N / m m 3]$

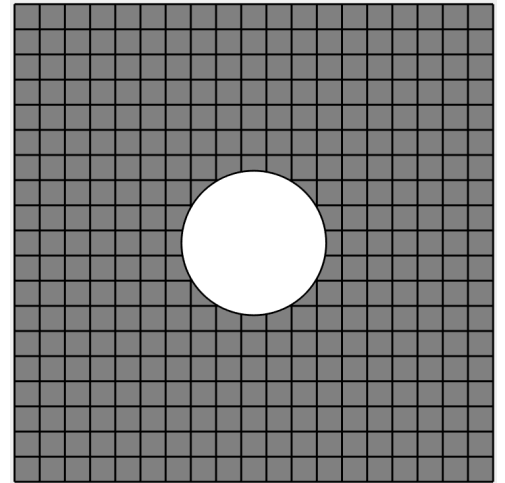

(a)

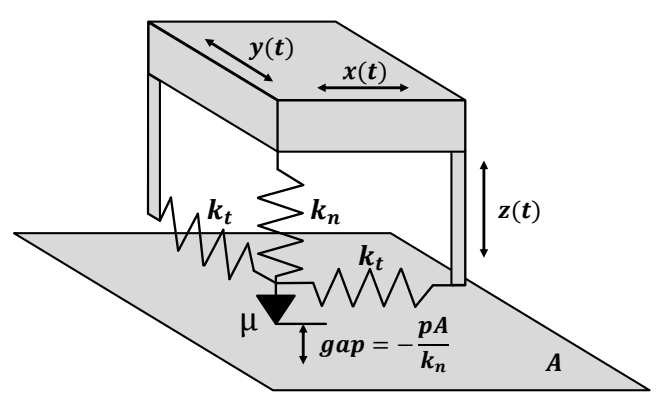

(b)

Figure 9: Nonlinear contact element locations (a) and 3D nonlinear contact element (b)

\subsection{Nonlinear dynamic response: comparison between the current modelling approach and the newly pro- posed multiscale approach}

Figure 11 shows the obtained dynamic response under different levels of excitation in the $x$ direction (see Fig. 6 which shows the excitation and response nodes location) for the baseline and the updated stiffness model, which illustrates the typical behaviour of a frictional joint: at low excitation level, the joint is fully stuck so its response is linear whereas at higher excitations, some parts of the joint interface start slipping, which leads to a softening effect and an increased damping. The presented results are consistent with the numerical and experimental results of Süß $[3,46]$.

Figure 11 also shows that the newly proposed multiscale approach leads to very similar results to the baseline model with constant contact stiffness across the contact area. This can be probably attributed to the closeness between the baseline and the average multiscale contact stiffness, which leads to a similar stiffness of the entire contact, and therefore to a similar frequency behaviour of the global dynamic response shown in Fig. 11.

Figure 12 shows the applied pressure on each nonlinear contact element as well as the resulting contact conditions and dissipated energy at resonance under an excitation of $500[N]$. Similar results are obtained with both methods: in the region underneath the bolt, the high pressure resulting from the tightening of the bolt prevents relative slip at the contact interface; this part of the joint remains fully stuck and therefore does not dissipated any energy. Outside this area, further away from the bolt, the pressure decreases and relative motion is observed; the nonlinear contact elements experience stick-slip transitions and dissipate frictional energy. Even further away from the bolt, at the edges of the contact area, the much lower pressure allows separation between the two contacting surfaces during a vibration cycle, leading to a mixture of energy dissipation and total loss of contact stiffness. 


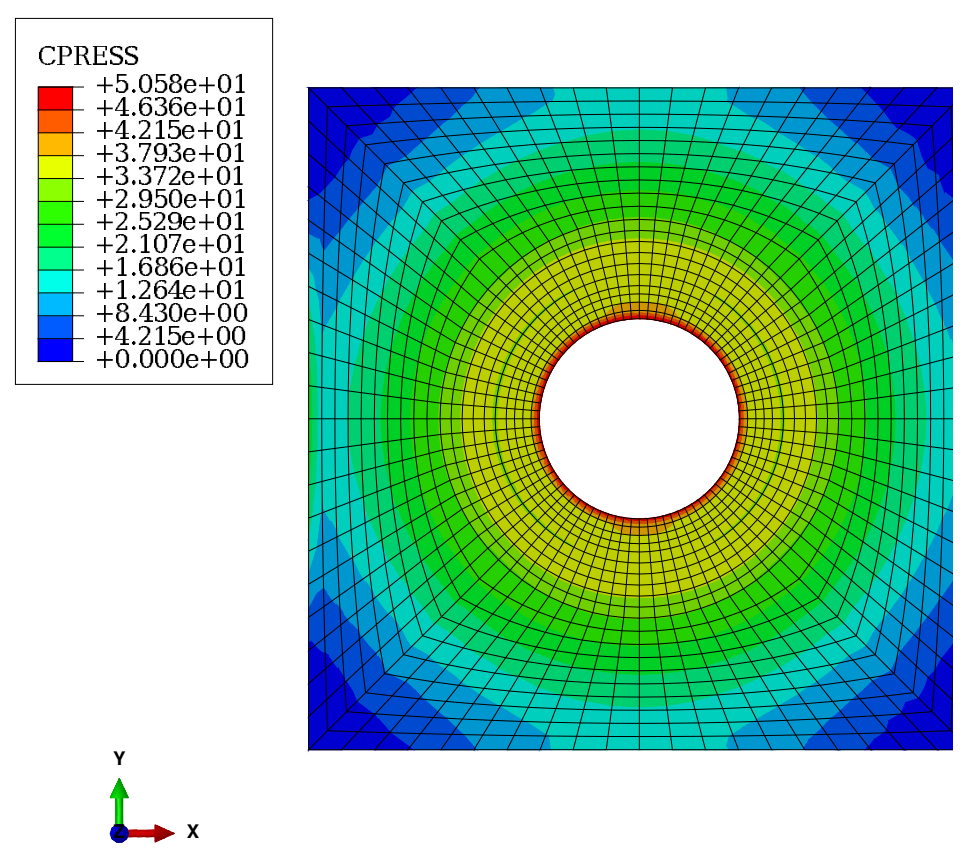

Figure 10: Contact pressure in $[\mathrm{MPa}]$ under a $30 \mathrm{kN}$ bolt tightening load

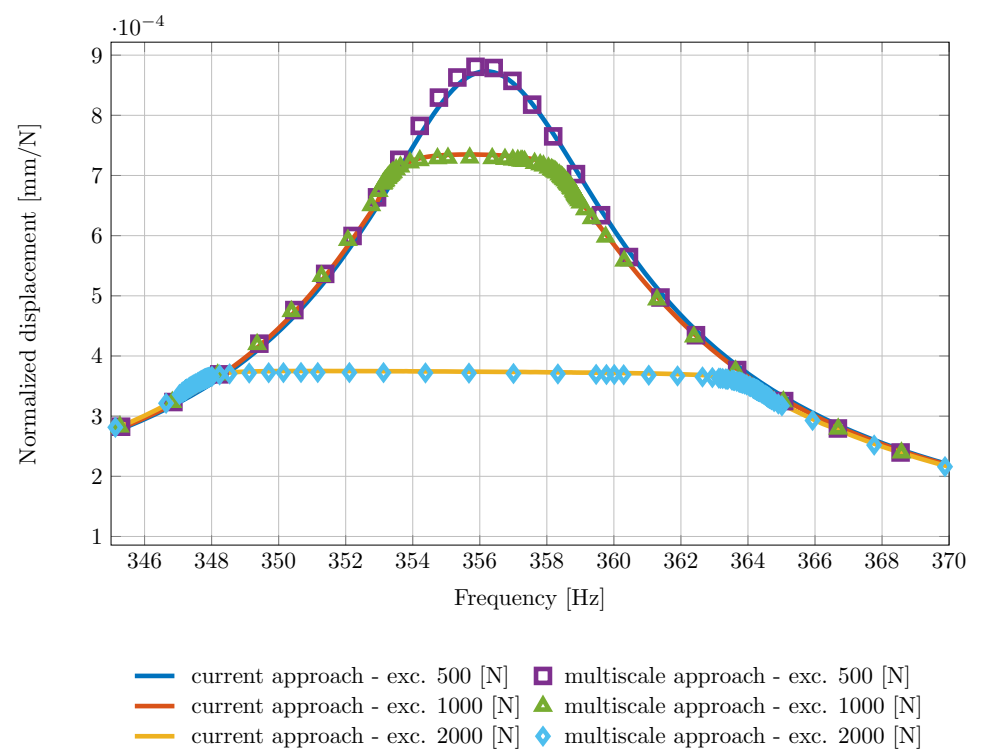

Figure 11: Frequency response functions of the resonator

It is worth noting that despite using the same normal load for each contact element within the two approaches, localised differences can be observed in the contact conditions and dissipated energy. These differences are due to the change of contact stiffness alone, i.e. using an uniform value of contact stiffness across the contact area for the baseline model and values computed for each friction element using the BEM-based contact solver for the newly proposed model. This demonstrates the potential of the proposed multiscale approach to capture the localised contact conditions accurately which is particularly important 
for future wear predictions or joint contact optimisation.

(a)
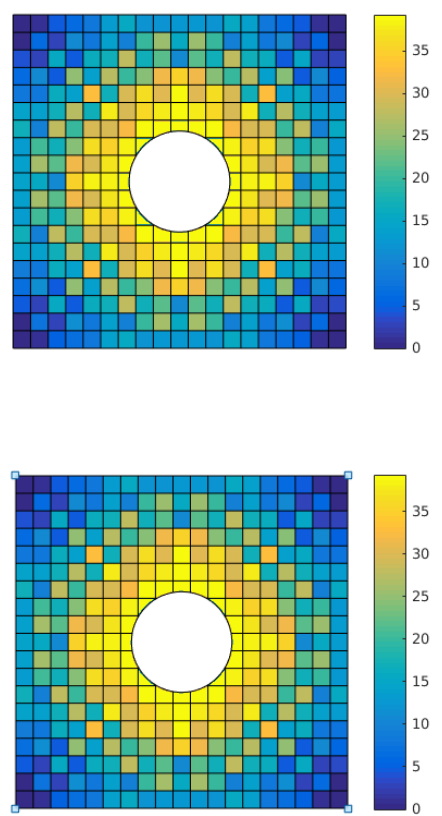

(b)

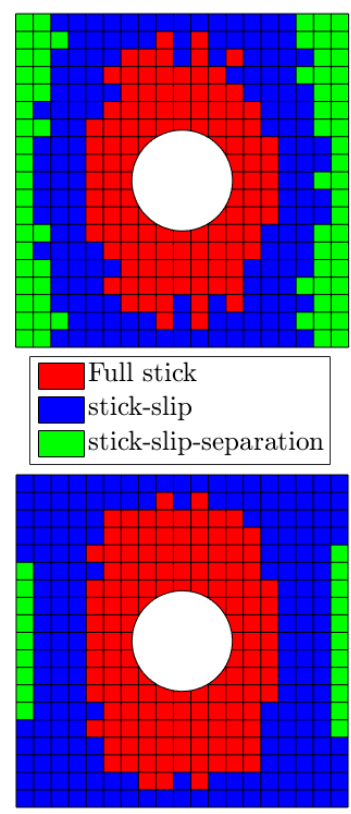

(c)
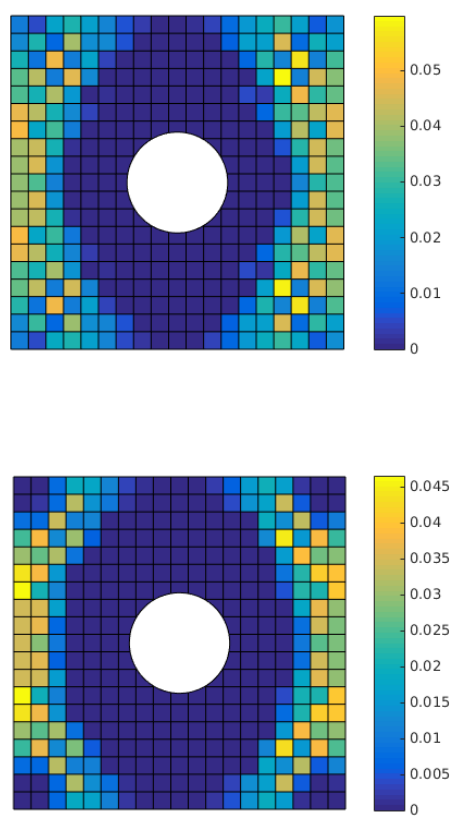

Figure 12: Contact pressure in [MPa] (a), contact conditions (b) and dissipated energy in [mJ] (c) at resonance for the reference (top) and the new model (bottom)

\section{Study of the effects of roughness on the nonlinear dynamic response}

Surface roughness is expected to strongly impact the normal and tangential contact stiffness and therefore the computation of the local contact conditions for a rough contact is likely to have much bigger impact on the nonlinear dynamic response than that observed for the previously considered smooth surface.

\subsection{Comparison between smooth and rough contact}

To investigate the effect of surface roughness, different surface finishes have been generated, where the RMS roughness was kept between $0.025[\mu \mathrm{m}]$ and $50[\mu \mathrm{m}]$, as given in ISO 1302. All these rough surfaces have been generated using $256 \times 256$ points. As a result, the analyses performed in the contact solver on the entire contact surface were done using a regular grid of $256 \times 256$ elements. A finer meshing of the surface in the contact solver would not increase the accuracy but instead would amplify the boundary effects at the edges of the contact area. Figure 13 shows one of the simulated rough profiles and the resulting contact morphology under a $30[k N]$ normal load. The heights of the profile follow a Gaussian distribution with a root-mean-square (RMS) roughness $R_{q}=10[\mu \mathrm{m}]$ and a correlation lengths of $0.5[\mathrm{~mm}]$ in both $x$ and $y$ directions. The heights of the profile have been magnified in Fig. 13 to allow the visualisation of the roughness. As can be seen on the right part of that figure, the real contact area is significantly reduced compared to the smooth surface and represents only $2.7 \%$ of the total potential contact area. This results in a completely different pressure distribution now consisting in local peaks of much high pressure.

Smaller values of $R_{q}(1[\mu \mathrm{m}]$ and $5[\mu \mathrm{m}])$ have also been generated and the corresponding results are shown in section 5.2 , but due to their similarity to the $R_{q}=10[\mu \mathrm{m}]$ case only the latter one will be discussed in detail. In order to evaluate the effect of the resulting localised pressure distribution on the dynamic response, initially a nonlinear dynamic analysis was performed using the new pressure distribution only, but keeping the reference values of contact stiffness, i.e. $k_{n}=k_{t}=6 e 4[\mathrm{~N} / \mathrm{mm} 3]$ for all nonlinear contact elements. In 

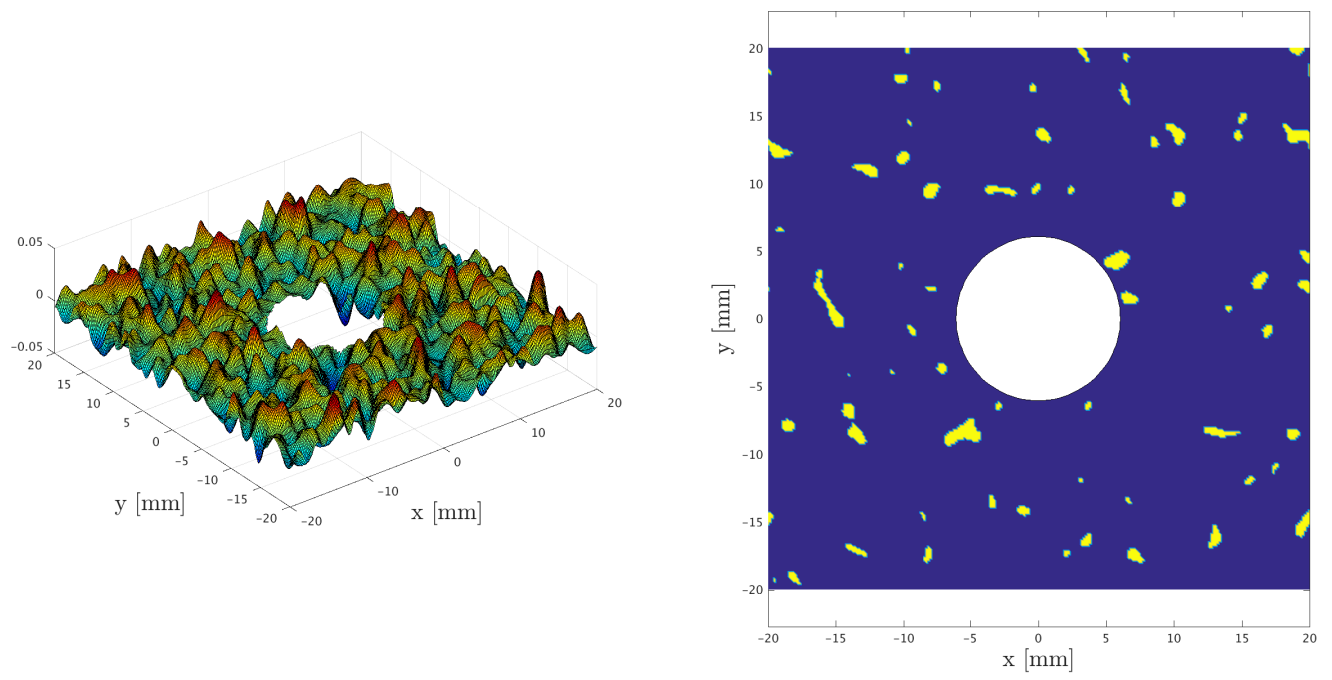

Figure 13: Profile heights [mm] (left) and morphology of the contact under a $30[k N]$ normal load (right)

a next step, the normal contact stiffnesses was computed with the contact solver, and proportional values of tangential contact stiffnesses were obtained by Eq. 6, since it was expected that the contact stiffness at the different elements would vary significantly. Finally, the values of $k_{n}$ and $k_{t}$, computed from the contact solver were used to consider all relevant effects for the dynamic response of the system. These three configurations will be referred to respectively as 'multiscale v1', 'multiscale v2' and 'multiscale v3' (see Tab. 1). In the analysis of each local contact area, for which contact stiffnesses have been extracted, a regular grid of $16 \times 16$ elements was used, which is enough to reproduce the local surface profile accurately. As mentioned for the global contact analysis, a further mesh refinement would amplify the boundary effects at the edges of each local contact element. The resulting distributions of stiffness computed by the contact solver are shown in Fig. 14. Fig. 14a) shows the normal contact stiffness used in the configurations 'multiscale v2' and 'multiscale v3'. Fig. 14b) is the tangential contact stiffness, proportional to the computed values of $k_{n}$, according to Eq. 6 and used in 'multiscale v2'. Finally, Fig. 14c) shows the computed tangential stiffness from the contact solver, used in 'multiscale v3'. As expected, the computed values of stiffness vary greatly across the contact area, in line with the non-uniform pressure distribution shown in Fig. 13.

In order to quantify the effects of the computed contact stiffnesses on the global dynamic response, an equivalent value was calculated for each configuration, representing an average value of stiffness between all nonlinear contact elements. It can be seen in Fig. 15 that despite using the reference value of $6 e 4$ $[N / m m 3]$ in 'multiscale v1', a significant drop in global joint stiffness occurs, since the roughness reduces the real contact area so that fewer nonlinear contact elements actually come into contact during the dynamic analysis. A further drop of the global joint stiffness is observed for the configurations 'multiscale v2' and 'multiscale v3'. This is due to the fact that all local contact stiffnesses computed by the contact solver are below $4.5 e 4[\mathrm{~N} / \mathrm{mm} 3]$ which is lower than the baseline value of $6 e 4[\mathrm{~N} / \mathrm{mm} 3]$. Interestingly, using the Mindlin analytical solution for the tangential contact stiffness (see Eq. 6) yields to almost the same equivalent global tangential stiffness than that obtained using the contact solver values. This means that in this particular case, Mindlin theory is providing a good average value of the tangential contact stiffness. However, some discrepancies can be observed locally in Fig. 14 which justifies the need to use the contact solver for an accurate evaluation of the local contact conditions.

The nonlinear dynamic response for the 1st breathing mode of the oscillator for each of the above mentioned joint models was computed at three different excitation levels $(500[N], 1000[N], 2000[N])$, and 


\begin{tabular}{l|c|c|c} 
& Normal load & $k_{n}$ & $k_{t}$ \\
\hline \hline current approach (smooth) & from Abaqus & $6 e 4[N / m m 3]$ & $6 e 4[N / m m 3]$ \\
multiscale v1 (rough) & from contact solver & $6 e 4[N / m m 3]$ & $6 e 4[N / m m 3]$ \\
multiscale v2 (rough) & from contact solver & from contact solver & $\frac{2(1-\nu)}{(2-\nu)} k_{n}$ \\
multiscale v3 (rough) & from contact solver & from contact solver & from contact solver \\
\hline \hline
\end{tabular}

Table 1: Contact parameters for all tested configurations

(a)

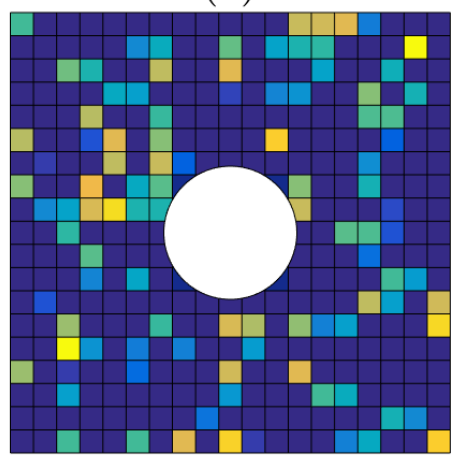

(b)

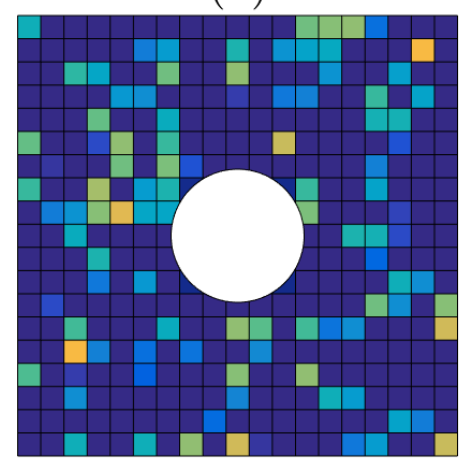

(c)

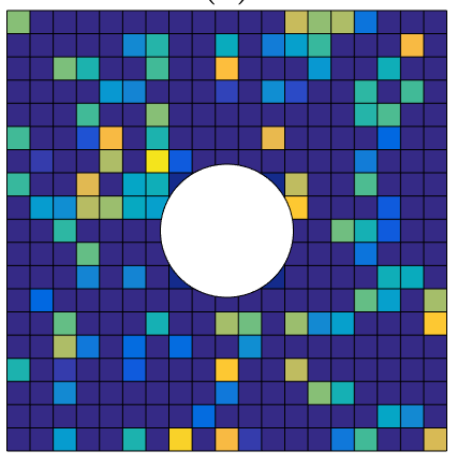

0

0.5

1

1.5

2

2.5

3

3.5

4

$\times 10^{4}$

Figure 14: (a) Computed normal contact stiffness $k_{n}[N / m m 3]$, (b) Mindlin tangential contact stiffness [N/mm3] and (c) computed $k_{t}[N / m m 3]$

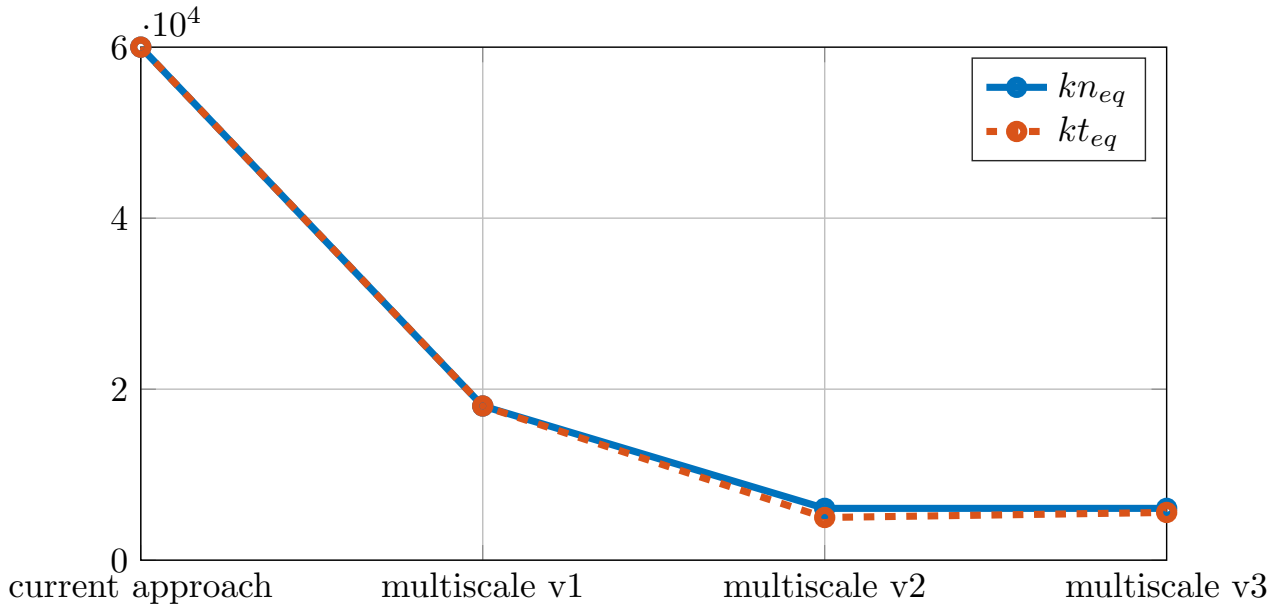

Figure 15: Equivalent normal and tangential contact stiffness for all tested configurations

the results are shown in Fig. 16. The changes of contact stiffness between the multiscale approaches v1 , v2 and v3 do not result in a significant changes of the global dynamic response which is in line with their relatively similar averaged values from Fig. 15. It is interesting to notice, that the baseline approach with 

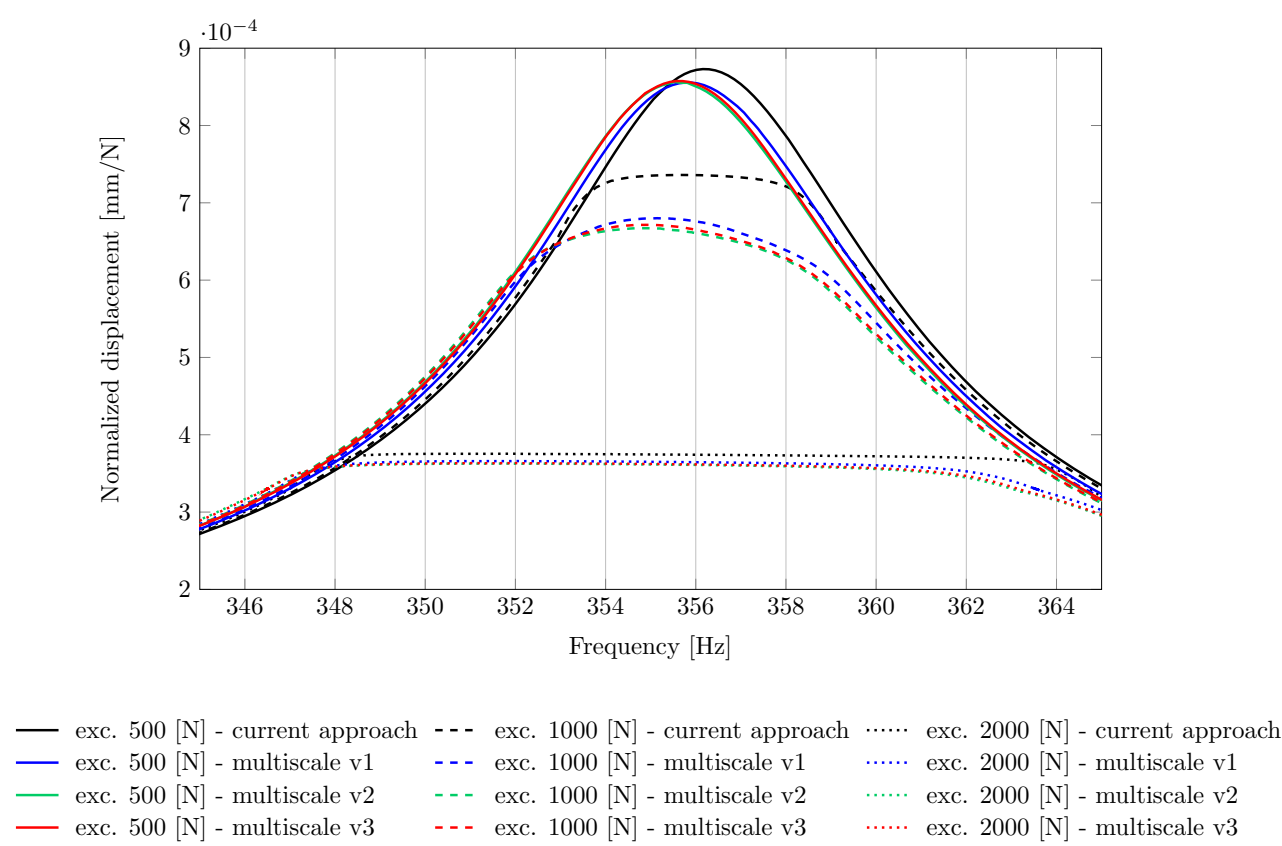

Figure 16: Frequency response functions of the resonator for different contact parameter setups
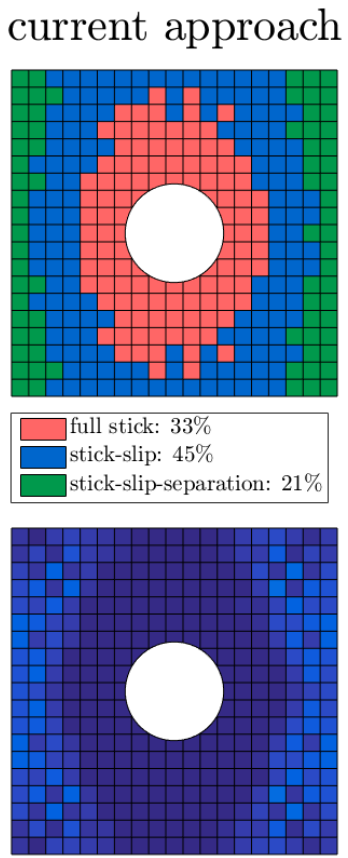

multiscale v1
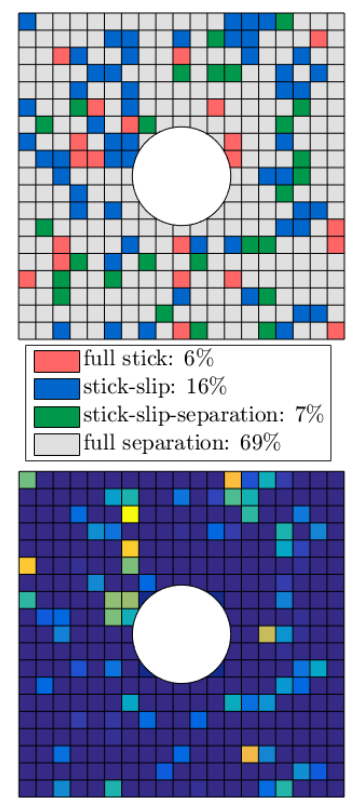

multiscale v2
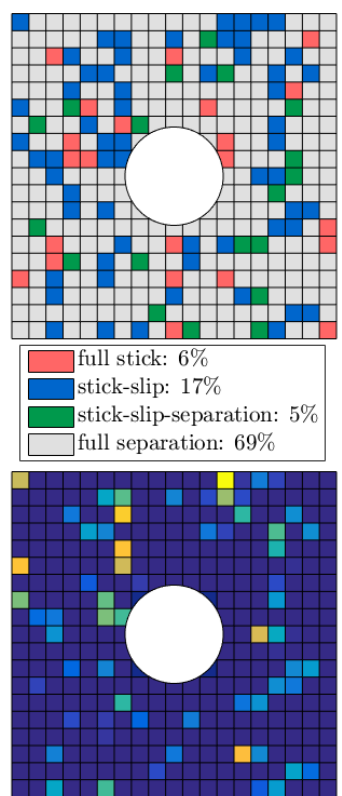

multiscale v3
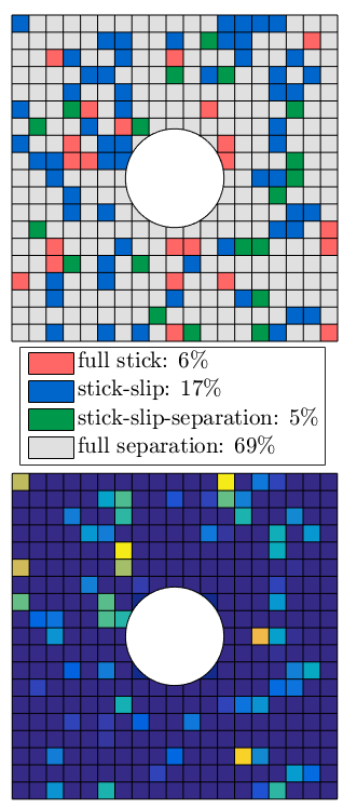

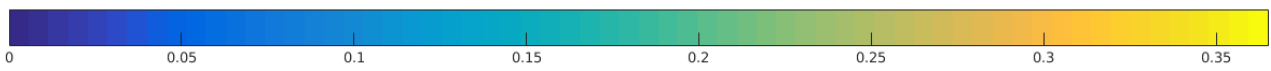

Figure 17: Contact conditions (top) and frictional dissipated energy distribution [mJ] (bottom) at resonance under a 500 [N] excitation

a much higher contact stiffness still leads to only marginally stiffer resonance frequencies, indicating that this particular joint geometry is relatively robust with regards to contact stiffness variations. All multiscale 


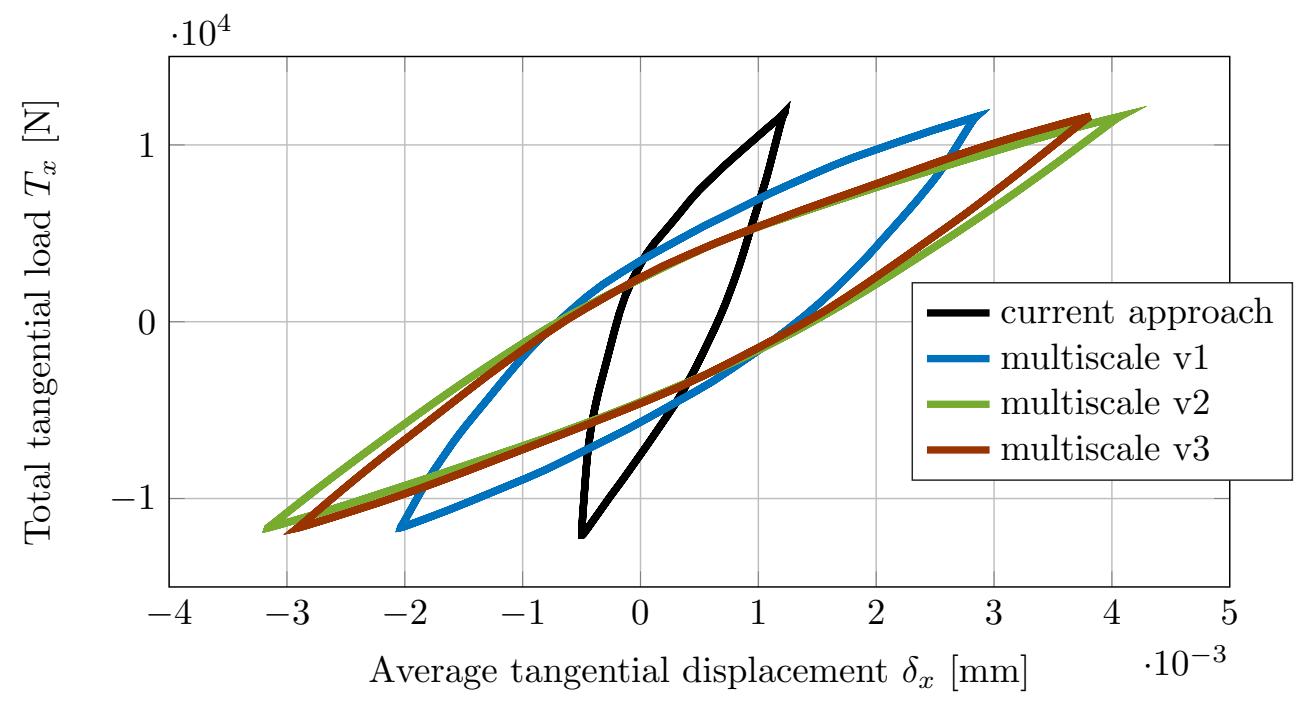

Figure 18: Frictional hysteresis loops at resonance under a $500[\mathrm{~N}]$ excitation

approaches tend to increase the energy dissipation in the contact, leading to a reduction in amplitude, but only the $1000[N]$ case shows a significant change. This is reflected in the local contact conditions and frictional dissipated energy at resonance in Fig. 17, where a very different picture emerges for the multiscale approach when compared to the baseline model. The originally clearly identifiable zones of stick, slip and separation have entirely disappeared, with all contact conditions now distributed over the entire contact zone. Although a reduced number of nonlinear elements is undergoing stick-slip transitions for the multiscale models the tangential relative motion between the contacting surfaces is much larger, which in turn leads to a larger frictional energy dissipation in certain hotspots (see bottom row of Fig. 17). To further demonstrate this increase in energy dissipation, the global hysteresis loop of a single vibration cycle for all four configurations has been plotted in Fig. 18, showing the significantly larger displacements and enclosed area of the multiscale approach.

\subsection{Effects of $R_{q}$ on the nonlinear dynamic response}

To understand the impact of different roughness values on the contact stiffness and the nonlinear dynamic response, four different surfaces with a root-mean-square roughness $R_{q}$ of $1 \mu \mathrm{m}, 5 \mu \mathrm{m}, 10 \mu \mathrm{m}$ and $15 \mu \mathrm{m}$ and correlation lengths $c l_{x}=c l_{y}=0.5 \mathrm{~mm}$ were generated. Figure 20 shows the resulting morphology of the contact where it can be seen that as $R_{q}$ increases, the fractional real area of contact decreases from $22.1 \%$ to $2.0 \%$. With regards to the resulting stiffness (computed following multiscale v3), as $R_{q}$ increases the real contact area decreases, leading to an increase of the local pressures and therefore to an increase of the corresponding local contact stiffnesses from the contact solver. Interestingly, Fig. 19 shows that the equivalent stiffness of the joint decreases for higher values of $R_{q}$, which can be attributed to the fact that the loss of contact stiffness due to the decrease of the real contact area has a much stronger impact on the global stiffness than the increase of some local contact stiffnesses.

The effect of the roughness values on the nonlinear frequency response can be seen in Figure 21 for the four different profiles and for three different excitation levels, $500[N], 1,000[N]$ and 2,000[N]. The same dependency on the $R_{q}$ values can be observed for all three excitation levels: the higher $R_{q}$, the lower the resonance frequency and the higher the energy dissipation. The drop in frequency is in line with the reduction in global stiffness at higher $R_{q}$ values observed in Fig. 19, while the change in energy dissipation can be explained with Fig. 22. It shows that for higher values of $R_{q}$, the same tangential load $T_{x}$ leads to higher relative tangential motions. As a result, although there are less and less contact elements dissipating energy, the total frictional dissipated energy, which is equal to the area of the presented hysteresis loops, 


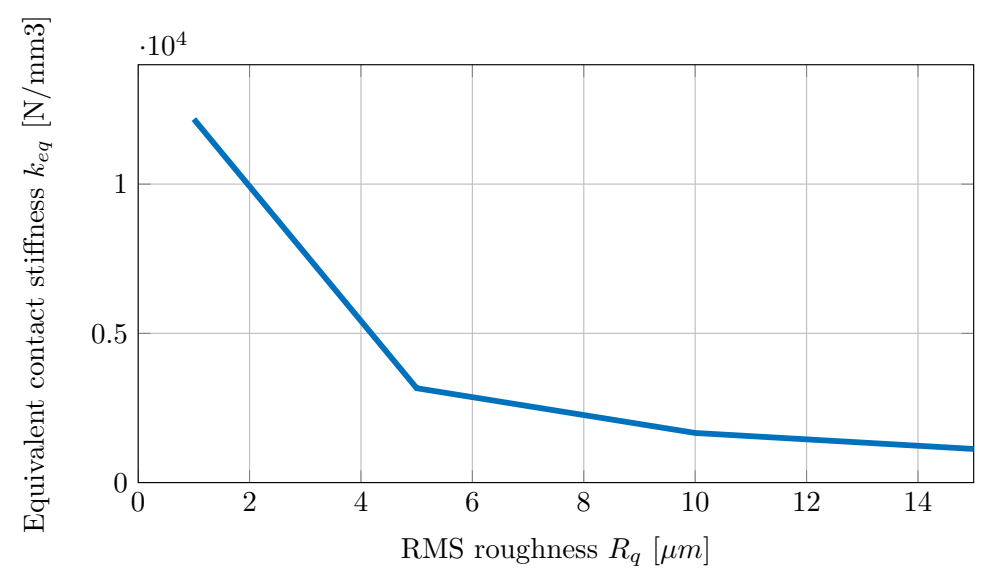

Figure 19: Equivalent contact stiffness $k_{e q}$ for different values of $R_{q}$
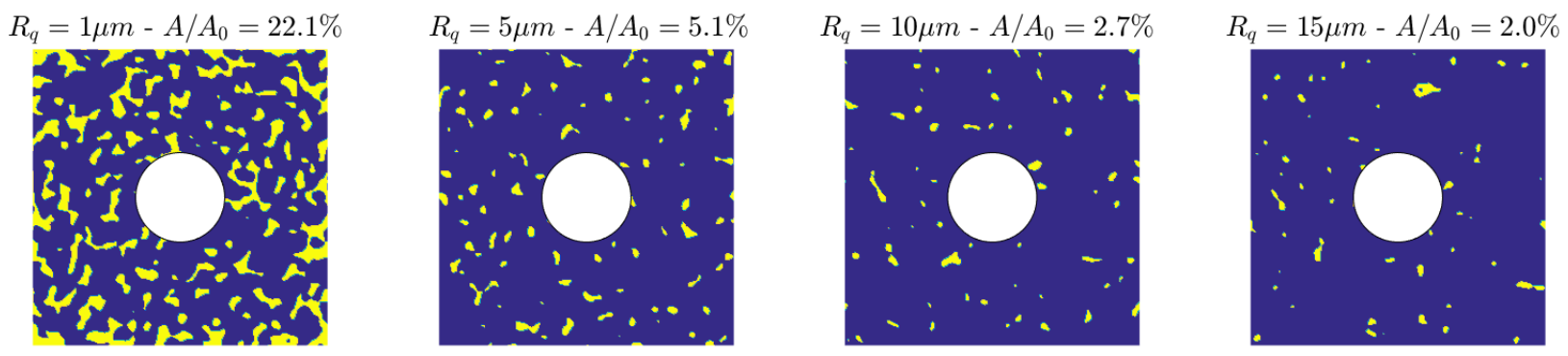

Figure 20: Morphology of the contact under a $30 \mathrm{kN}$ normal load for different values of RMS roughness
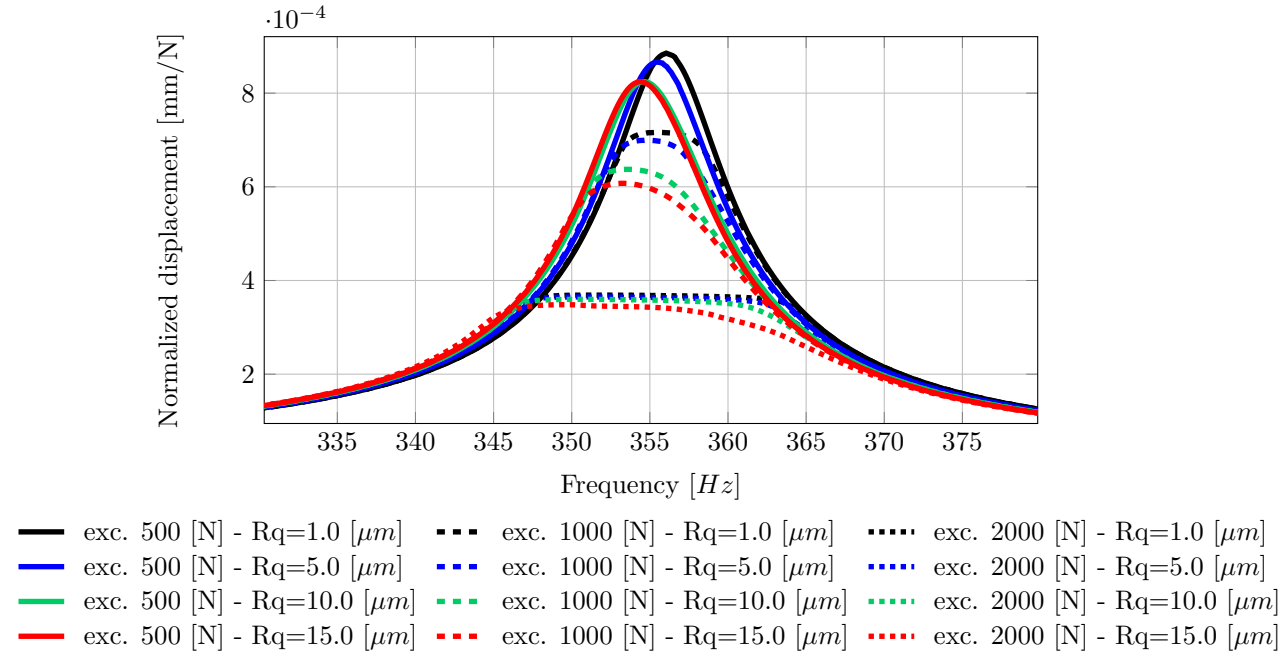

Figure 21: Frequency response functions of the resonator for different values of $R_{q}$

increases with $R_{q}$, leading to a more damped frequency response function. 


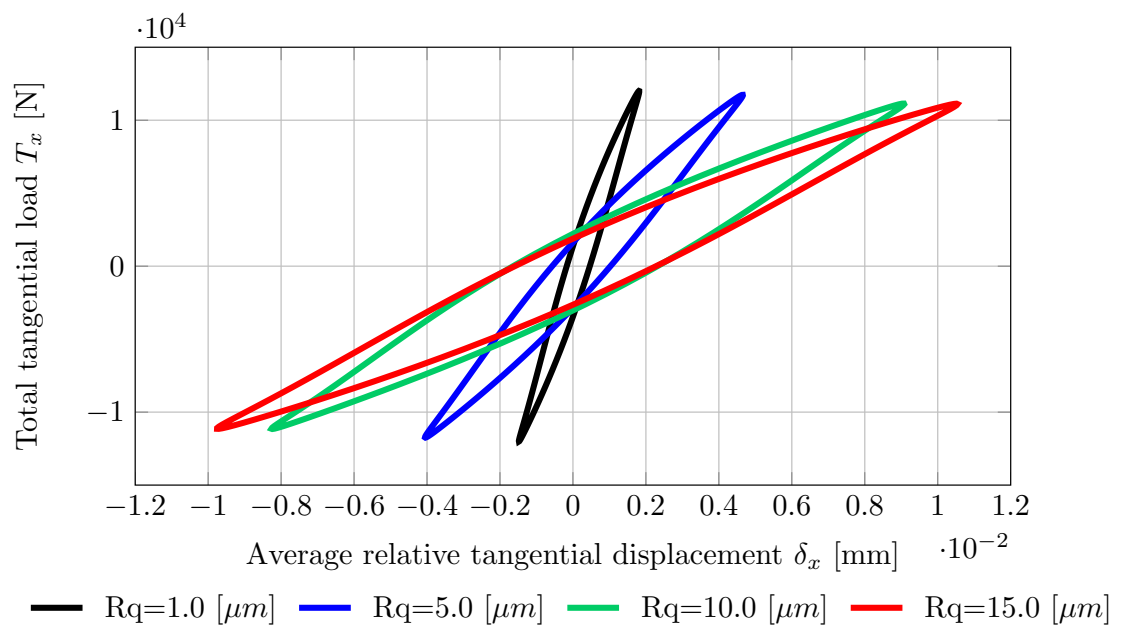

Figure 22: Frictional hysteresis loops at resonance for different values of $R_{q}$ under 500 [N] excitation

\section{Discussions}

\subsection{Comments on the proposed approach and its results}

The proposed approach permitted to evaluate the influence of roughness on the nonlinear dynamic response of a friction joint. It was shown that the surface roughness has a very strong impact on the contact conditions that are the pressure distribution and the normal and tangential contact stiffnesses. Roughness reduces drastically the real contact area, which leads to a fundamentally different contact pressure than that of a smooth surface; very localised peaks of pressure appear and lead to higher values of local normal contact stiffness. At the same time, the decrease of the real contact area leads to fewer active friction elements and therefore to a decrease of the global stiffness of the joint. This last mechanism was shown to have a stronger impact than the increase of local contact stiffness since the global stiffness of the joint was observed to decrease with roughness. The same observations were made on the tangential contact stiffness. It was also observed that the Mindlin analytical solution was providing a good average value of the tangential contact stiffness, so it could potentially be used to save computational time if only the global dynamic behaviour of the joint is of interest. By computing the frequency response functions of the first breathing mode of the joint, it was shown that the newly proposed approach only led to small variations of resonance frequency and energy dissipation. However, this does not mean that the baseline approach should not evolve since the small differences can be explained by the fact that the computed global joint stiffness, which drives the frequency response function that represents the global behaviour of the joint, happened to be close to the reference value of $6 e 4[\mathrm{~N} / \mathrm{mm} 3]$, which could be different for another application. Furthermore, larger differences between the baseline approach and the newly proposed approach were observed on the local contact conditions and in particular completely different energy dissipation mechanisms were observed with the new approach. These local phenomena resulting from surface roughness, which are critical if it is intended to optimise the joint design or to predict wear, can only be captured with the proposed approach. It is worth reminding that for even more accurate evaluation of the contact conditions with regards to roughness, plasticity should be considered.

\subsection{Computational cost of the proposed approach}

In order to provide the reader with an estimate of the computational cost of the proposed approach, the wall clock times corresponding to some of the presented simulations have been provided. Table 2, shows the computational time that was necessary to obtain one curve of the nonlinear frequency responses given in Fig. 16 and Fig. 21 under 1000[N] excitation. The simulations were run on a HPC node with a 20-core CPU (Intel Xeon E5-2620, 2GHz). It can be seen that the simulations with rough contact required only 
half the time to run. Due to the roughness, the real contact area is significantly reduced, which means that fewer nonlinear friction elements will be active during the simulation, and therefore the computational cost is reduced. Similarly the computational cost is higher for smaller values of roughness.

\begin{tabular}{c|c|c|c|c|c} 
& Smooth & $R_{q}=1.0[\mu \mathrm{m}]$ & $R_{q}=5.0[\mu \mathrm{m}]$ & $R_{q}=10.0[\mu \mathrm{m}]$ & $R_{q}=15.0[\mu \mathrm{m}]$ \\
\hline \hline Wall clock time $[\mathrm{s}]$ & 13682 & 6957 & 6362 & 6031 & 5954 \\
\hline \hline
\end{tabular}

Table 2: Computational cost of nonlinear dynamic analysis in [s] for smooth and rough surfaces

The other contributor to the computational cost of the proposed approach is the contact analysis performed using the BEM-based contact solver. Table 3 gives the computational time necessary to solve (i) the normal contact problem, i.e. extract the normal load $N_{0}$ and the normal contact stiffness $k_{n}$, and (ii) the tangential contact problem which includes the computation of the frictional hysteresis loop and extracting the tangential contact stiffness $k_{t}$, for all contact elements. The contact solver simulations were run using one CPU of a laptop Intel Core i7-4700HQ 2.40GHz. Similarly to what was observed for the nonlinear dynamic analysis, it can be seen that higher values of RMS roughness lead to a shorter computational time.

\begin{tabular}{l|c|c|c|c} 
& $R_{q}=1.0[\mu \mathrm{m}]$ & $R_{q}=5.0[\mu \mathrm{m}]$ & $R_{q}=10.0[\mu \mathrm{m}]$ & $R_{q}=15.0[\mu \mathrm{m}]$ \\
\hline \hline Normal contact & 642 & 305 & 171 & 150 \\
\hline Tangential contact & 9969 & 4527 & 2105 & 1821 \\
\hline \hline
\end{tabular}

Table 3: Computational cost of contact analysis in $[\mathrm{s}]$ for different roughness values

Based on the presented figures and provided that some parallel computing is used for the contact analysis, it can be claimed that the computational cost of the proposed approach could end up being lower or roughly the same as the one of the current approach. In any case, it was shown that the gain of accuracy of the proposed approach does not come at a prohibitive computational cost.

\section{Conclusions}

In this paper, a multiscale approach was introduced to evaluate the effects of surface roughness on the nonlinear dynamic response of a bolted joint. The approach successfully coupled a multiharmonic balance solver to a recently developed and updated contact solver based on the Boundary Element Method, where the latter was used for a refined contact analysis and a more accurate evaluation of the local contact parameters for the nonlinear dynamic analysis.

The presented results highlight the sensitivity of the nonlinear dynamic response to changes at the contact interface; the surface roughness reduces drastically the real contact area, which leads to a complete redistribution of the contact pressure and to a strongly non-uniform distribution of contact stiffness. It could be shown that for low values of RMS roughness (below $5 \mu \mathrm{m}$ ), the effects of the roughness on the global nonlinear dynamic response is small enough to be neglected in the analysis and even for larger values the global effect is relatively small and smeared values can provide a reasonably good prediction.

However, if it is intended to obtain accurate local contact predictions in order to optimise the joint design to maximize energy dissipation or to predict wear, then the effects of roughness must be considered in the analysis. Expectedly, this gain of accuracy comes at an extra computational cost, but this cost was shown to be acceptable. For higher values of RMS roughness, both the global and local behaviour of the joint are significantly affected.

\section{Acknowledgements}

The authors are grateful to Innovate UK and Rolls-Royce plc for providing the financial support for this work and for giving permission to publish it. This work is part of a collaborative R\&T project 'SILOET II 
Project 10' which is co-funded by Innovate UK and Rolls-Royce plc and carried out by Rolls-Royce plc and the Vibration UTC at Imperial College London.

\section{References}

[1] L. Salles, C. Swacek, R. M. Lacayo, P. Reuss, M. R. W. Brake, C. W. Schwingshackl, Numerical Round Robin for Prediction of Dissipation in Lap Joints, Springer, 2016, pp. 53-64. doi:10.1007/978-3-319-15221-94.

[2] J. Gross, J. Armand, R. M. Lacayo, P. Reuß, L. Salles, C. W. Schwingshackl, M. R. W. Brake, R. J. Kuether, A numerical round robin for the prediction of the dynamics of jointed structures, Springer, 2016, pp. 195-211. doi:10.1007/978-3-319-15221-936.

[3] D. Süß, K. Willner, Investigation of a jointed friction oscillator using the multiharmonic balance method, Mechanical Systems and Signal Processing 52-53 (2015) 73-87. doi:10.1016/j.ymssp.2014.08.003.

[4] M. Eriten, M. Kurt, G. Luo, D. M. McFarland, L. A. Bergman, A. F. Vakakis, Nonlinear system identification of frictional effects in a beam with a bolted joint connection, Mechanical Systems and Signal Processing 39 (1-2) (2013) 245-264. doi:10.1016/j.ymssp.2013.03.003.

[5] A. A. Ferri, Friction damping and isolation systems, Journal of Mechanical Design 117 (B) (1995) 196. doi:10.1115/1.2836456.

[6] L. Gaul, J. Lenz, Nonlinear dynamics of structures assembled by bolted joints, Acta Mechanica 125 (1-4) (1997) 169-181. doi:10.1007/bf01177306.

[7] C. Hartwigsen, Y. Song, D. McFarland, L. Bergman, A. Vakakis, Experimental study of non-linear effects in a typical shear lap joint configuration, Journal of Sound and Vibration 277 (1-2) (2004) 327-351. doi:10.1016/j.jsv.2003.09.018.

[8] K.-H. Koh, J. H. Griffin, S. Filippi, A. Akay, Characterization of turbine blade friction dampers, Journal of Engineering for Gas Turbines and Power 127 (4) (2005) 856. doi:10.1115/1.1926312.

[9] P. Gray, C. McCarthy, A global bolted joint model for finite element analysis of load distributions in multi-bolt composite joints, Composites Part B: Engineering 41 (4) (2010) 317-325. doi:10.1016/j.compositesb.2010.03.001.

[10] J. Kim, J.-C. Yoon, B.-S. Kang, Finite element analysis and modeling of structure with bolted joints, Applied Mathematical Modelling 31 (5) (2007) 895-911. doi:10.1016/j.apm.2006.03.020.

[11] J. D. Reid, N. R. Hiser, Detailed modeling of bolted joints with slippage, Finite Elements in Analysis and Design 41 (6) (2005) 547-562. doi:10.1016/j.finel.2004.10.001.

[12] V. Kaliakin, J. Li, Insight into deficiencies associated with commonly used zero-thickness interface elements, Computers and Geotechnics 17 (2) (1995) 225-252. doi:10.1016/0266-352x(95)93870-o.

[13] C. S. Desai, M. M. Zaman, J. G. Lightner, H. J. Siriwardane, Thin-layer element for interfaces and joints, International Journal for Numerical and Analytical Methods in Geomechanics 8 (1) (1984) 19-43. doi:10.1002/nag.1610080103.

[14] M. Mayer, L. Gaul, Segment-to-segment contact elements for modelling joint interfaces in finite element analysis, Mechanical Systems and Signal Processing 21 (2) (2007) 724-734. doi:10.1016/j.ymssp.2005.10.006.

[15] K. G. Sharma, C. S. Desai, Analysis and implementation of thin-layer element for interfaces and joints, Journal of Engineering Mechanics 118 (12) (1992) 2442-2462. doi:10.1061/(asce)0733-9399(1992)118:12(2442).

[16] J. H. Griffin, Friction damping of resonant stresses in gas turbine engine airfoils, Journal of Engineering for Power 102 (2) (1980) 329. doi:10.1115/1.3230256.

[17] K. Y. Sanliturk, D. J. Ewins, A. B. Stanbridge, Underplatform dampers for turbine blades: Theoretical modeling, analysis, and comparison with experimental data, J. Eng. Gas Turb. Power 123 (4) (2001) 919. doi:10.1115/1.1385830.

[18] D. J. Segalman, Modelling joint friction in structural dynamics, Structural Control and Health Monitoring 13 (1) (2006) 430-453. doi:10.1002/stc.119.

[19] W. D. Iwan, A distributed-element model for hysteresis and its steady-state dynamic response, Journal of Applied Mechanics 33 (4) (1966) 893. doi:10.1115/1.3625199.

[20] C. W. Schwingshackl, D. D. Maio, I. Sever, J. S. Green, Modeling and validation of the nonlinear dynamic behavior of bolted flange joints, Journal of Engineering for Gas Turbines and Power 135 (12) (2013) 122504. doi:10.1115/1.4025076.

[21] E. P. Petrov, D. J. Ewins, State-of-the-art dynamic analysis for non-linear gas turbine structures, Proceedings of the Institution of Mechanical Engineers, Part G: Journal of Aerospace Engineering 218 (3) (2004) 199-211. doi:10.1243/0954410041872906.

[22] C. M. Firrone, S. Zucca, M. M. Gola, The effect of underplatform dampers on the forced response of bladed disks by a coupled static/dynamic harmonic balance method, International Journal of Non-Linear Mechanics 46 (2) (2011) 363-375. doi:10.1016/j.ijnonlinmec.2010.10.001

[23] E. Cigeroglu, N. An, C.-H. Menq, Forced response prediction of constrained and unconstrained structures coupled through frictional contacts, Journal of Engineering for Gas Turbines and Power 131 (2) (2009) 022505. doi:10.1115/1.2940356.

[24] D. Charleux, C. Gibert, F. Thouverez, J. Dupeux, Numerical and experimental study of friction damping blade attachments of rotating bladed disks, International Journal of Rotating Machinery 2006 (2006) 1-13. doi:10.1155/ijrm/2006/71302.

[25] X. Shi, A. A. Polycarpou, Measurement and modeling of normal contact stiffness and contact damping at the meso scale, Journal of Vibration and Acoustics 127 (1) (2005) 52. doi:10.1115/1.1857920.

[26] S. Medina, D. Nowell, D. Dini, Analytical and numerical models for tangential stiffness of rough elastic contacts, Tribology Letters 49 (1) (2012) 103-115. doi:10.1007/s11249-012-0049-y.

[27] C. W. Schwingshackl, E. P. Petrov, D. J. Ewins, Effects of contact interface parameters on vibration of turbine bladed disks with underplatform dampers, Journal of Engineering for Gas Turbines and Power 134 (3) (2012) 032507. doi:10.1115/1.4004721.

[28] L. Pesaresi, L. Salles, A. Jones, J. Green, C. Schwingshackl, Modelling the nonlinear behaviour of an underplatform damper test rig for turbine applications, Mechanical Systems and Signal Processing 85 (2017) 662-679. doi:10.1016/j.ymssp.2016.09.007.

[29] E. P. Petrov, D. J. Ewins, Generic friction models for time-domain vibration analysis of bladed disks, J. Turbomach. 126 (1) (2004) 184. doi:10.1115/1.1644557. 
[30] C. W. Schwingshackl, E. P. Petrov, D. J. Ewins, Measured and estimated friction interface parameters in a nonlinear dynamic analysis, Mechanical Systems and Signal Processing 28 (2012) 574-584. doi:10.1016/j.ymssp.2011.10.005.

[31] E. P. Petrov, D. J. Ewins, Analytical formulation of friction interface elements for analysis of nonlinear multi-harmonic vibrations of bladed disks, J. Turbomach. 125 (2) (2003) 364. doi:10.1115/1.1539868.

[32] E. P. Petrov, A high-accuracy model reduction for analysis of nonlinear vibrations in structures with contact interfaces, J. Eng. Gas Turb. Power 133 (10) (2011) 102503. doi:10.1115/1.4002810.

[33] E. P. Petrov, D. J. Ewins, Advanced modeling of underplatform friction dampers for analysis of bladed disk vibration, J. Turbomach. 129 (1) (2007) 143. doi:10.1115/1.2372775.

[34] L. Gaul, R. Nitsche, The role of friction in mechanical joints, Applied Mechanics Reviews 54 (2) (2001) 93. doi:10.1115/1.3097294.

[35] D. Hills, D. Nowell, Mechanics of fretting fatigueoxford's contribution, Tribology International 76 (2014) 1 - 5, proceedings of the Seventh International Symposium on Fretting Fatigue. doi:http://dx.doi.org/10.1016/j.triboint.2013.09.015. URL http://www.sciencedirect.com/science/article/pii/S0301679X13003125

[36] S. Filippi, E. B. Rodrigues, M. M. Gola, , in: Volume 5: Marine Microturbines and Small Turbomachinery Oil and Gas Applications Structures and Dynamics, Parts A and B, ASME, 2006. doi:10.1115/gt2006-90757. URL

[37] C. W. Schwingshackl, Measurement of friction contact parameters for nonlinear dynamic analysis, in: Topics in Modal Analysis I, Volume 5, Springer Science Business Media, 2012, pp. 167-177.

[38] L. Salles, L. Blanc, F. Thouverez, A. M. Gouskov, Dynamic analysis of fretting-wear in friction contact interfaces, International Journal of Solids and Structures 48 (10) (2011) 1513-1524. doi:10.1016/j.ijsolstr.2011.01.035.

[39] L. Salles, L. Blanc, F. Thouverez, A. M. Gouskov, P. Jean, Dual time stepping algorithms with the high order harmonic balance method for contact interfaces with fretting-wear, J. Eng. Gas Turb. Power 134 (3) (2012) 032503. doi:10.1115/1.4004236.

[40] J. Armand, L. Salles, C. Schwingshackl, Numerical simulation of partial slip contact using a semi-analytical method, in: ASME 2015 International Design Engineering Technical Conferences and Computers and Information in Engineering Conference, American Society of Mechanical Engineers, 2015, pp. V008T13A022-V008T13A022.

[41] J. Armand, L. Pesaresi, L. Salles, C. Schwingshackl, A multiscale approach for nonlinear dynamic response predictions with fretting wear, Journal of Engineering for Gas Turbines and Power 139 (2) (2017) 022505.

[42] I. A. Polonsky, L. M. Keer, Fast methods for solving rough contact problems: A comparative study, J. Tribol. 122 (1) (2000) 36. doi:10.1115/1.555326.

[43] K. L. Johnson, Contact Mechanics, Cambridge University Press, 1985, cambridge Books Online.

[44] A. E. H. Love, A Treatise on the Mathematical Theory of Elasticity, A Treatise on the Mathematical Theory of Elasticity, at the University Press, 1906.

[45] R. D. Mindlin, Compliance of elastic bodies in contact, Trans.ASME, Series E, Journal of Applied Mechanics 16 (1949) $259-268$.

[46] D. Süß, Multi-harmonische-balance-methoden zur untersuchung des übertragungsverhaltens von strukturen mit fügestellen, Ph.D. thesis, Dissertation, Erlangen, Friedrich-Alexander-Universität Erlangen-Nürnberg (FAU), 2016 (2016). 